\title{
Prognostic value of c-Met expression in oral squamous cell carcinoma
}

\author{
CHRISTIAN FREUDLSPERGER, DOROTHEA ALEXANDER, SIEGMAR REINERT and JUERGEN HOFFMANN
}

\author{
Department of Oral and Maxillofacial Surgery, Tuebingen University Hospital, D-72076 Tuebingen, Germany
}

Received September 7, 2009; Accepted October 26, 2009

DOI: 10.3892/etm_00000012

\begin{abstract}
Expression of c-Met, an oncogene encoding the receptor for the hepatocyte growth factor, was found to be associated with the progression of various types of human carcinomas including squamous cell carcinoma of the head and neck. This study was performed to analyze the prognostic role of c-Met in oral squamous cell carcinoma (OSCC) by detecting its expression in relation to clinicopathological features. Two hundred and eleven patients with OSCC were analyzed for c-Met expression by immunohistochemistry. Specimens with $\geq 50 \%$ of cancer cells showing staining for c-Met were considered c-Met-positive. A pairwise multiple-comparison procedure (Fisher's exact test) and survival analyses using the Kaplan-Meier method were used to analyze the data. One hundred and seventy-five patients (82.9\%) showed positivity for c-Met, while 36 patients (17.1\%) showed no immunopositivity. In contrast to published studies, there was no statistically significant correlation between c-Met expression and tumor size, lymph node status, distant metastasis or survival rates. However, a statistically significant negative correlation between c-Met expression and tumor stage was apparent $(\mathrm{p}=0.022)$. Hence, this study did not confirm the role of c-Met as a prognostic marker in patients with OSCC.
\end{abstract}

\section{Introduction}

Oral squamous cell carcinoma (OSCC) is the most common epithelial oral cancer and is characterized by a high infiltrative potential, which can result in early regional lymph node involvement and subsequent metastatic spread. Despite advances in multidisciplinary treatment modalities, including chemotherapy and/or radiotherapy, no improvement in the 5 -year survival rate has been achieved over the past 20 years (1). In addition, the clinical behavior of OSCC is difficult to

Correspondence to: Dr Christian Freudlsperger, Department of Oral and Maxillofacial Surgery, Tuebingen University Hospital, Osianderstrasse 2-8, D-72076 Tuebingen, Germany

E-mail: christian.freudlsperger@klinikum.uni-tuebingen.de

Key words: oral squamous cell carcinoma, lymph node metastasis c-Met, hepatocyte growth factor, survival, prognosis predict, considering classical histopathological parameters only.

Thus, understanding the molecular mechanisms of local invasion has become an important goal in the field of head and neck surgery. Several reports have indicated that local interactions between cancer cells and stromal cells are important for the process of invasion and metastasis, including cellular detachment, proteolytic degradation of the basal lamina, migration through the extracellular matrix and resistance of apoptosis (2).

A proto-oncogene which has been suggested to be associated with OSCC progression is c-Met (3). c-Met encodes the receptor for hepatocyte growth factor (HGF). This receptor is a $190-\mathrm{kDa}$ receptor-like tyrosine kinase, comprised of disulfide-linked subunits $50(\alpha)$ and $145 \mathrm{kDa}$ ( $\beta$ ) (4). In the fully processed c-Met product, the $\alpha$-subunit is completely extracellular and the $\beta$-subunit has an extracellular ligand-binding domain, a single transmembrane domain and a cytoplasmic tyrosine kinase domain (4). c-Met is found in hepatocytes, fibrocytes, keratinocytes and melanocytes and also in the kidney, lung, spleen, hematopoietic cells and ovaries (4). c-Met is activated by HGF, a 100-kDa heparinbinding polypeptide, which is predominantly expressed by mesenchymal cells (5). HGF is structurally related to the family of serine proteases including plasminogen, prothrombin, urokinase and tissue plasminogen activator (6).

The HGF/c-Met interaction can promote cell proliferation, motility, invasiveness, morphogenesis and angiogenesis in various cell types, including different tumor cells $(6,7)$. In several clinical studies, high expression of HGF and c-Met has been shown in various types of human carcinomas including thyroid, pancreas, prostate and head and neck cancers (8), and its expression has been associated with poor prognosis in nasopharyngeal, esophageal, cervical and breast carcinoma (9). Furthermore, the expression of c-Met in head and neck squamous cell carcinoma (HNSCC), the clinicopathological parameters and the survival of HNSCC patients have been analyzed in various studies $(3,10,11)$, showing inconsistent results in terms of the prognostic value of c-Met expression in HNSCC. Therefore, the aim of the present study was to investigate the expression of c-Met in 211 patients with OSCC using immunohistochemistry and to analyze its expression in relation to clinicopathological parameters, in order to verify its role in the prognostic evaluation of these tumors. 


\section{Materials and methods}

Specimens for immunohistochemistry. For immunohistochemical evaluation, the routinely processed paraffin blocks of formalin-fixed OSCC specimens were obtained from 53 female and 158 male patients who underwent surgery at the Department of Oral and Maxillofacial Surgery of the University Hospital Tuebingen, Germany, between 1988 and 1997. The specimens were retrieved retrospectively from the pathology archives. The criteria for inclusion in this study included a histopathological diagnosis of OSCC, no preoperative therapy, potentially curative radical resection, follow-up data available and satisfactory tissue preservation.

The mean age of included patients was $58 \pm \mathrm{SD}$ years (range, 34-93 years). The follow-up period for surviving patients ranged from 1 to 172 months, with a median of 42 months. Primary tumor sites were as follows: maxilla, including soft and hard palate, 13 tumors; floor of the mouth, 79 tumors; tongue, 36 tumors; gingival of the mandible, 22 tumors; retromolar region, 8 tumors; lip, 15 tumors; planum buccale, 17 tumors; all other locations, 21 tumors. The TNM staging categories were determined according to the criteria established by the American Joint Committee on Cancer and the International Union Against Cancer (UICC) (12). Stage grouping of the patients was as follows: stage I, 47 patients; stage II, 30 patients; stage III, 27 patients; stage IV, 107 patients. On histopathologic evaluation of lymph node involvement, 146 tumors were classified as $\mathrm{N}_{0}$ and 65 tumors were classified as $\mathrm{N}_{1}$ or $\mathrm{N}_{2}$. One hundred and thirty-five tumors were $\leq 4 \mathrm{~cm}$ in diameter $\left(\mathrm{T}_{1}\right.$ and $\left.\mathrm{T}_{2}\right)$ and 76 tumors were $>4 \mathrm{~cm}$ in diameter $\left(\mathrm{T}_{3}\right.$ and $\left.\mathrm{T}_{4}\right)$. Two tumors showed distant metastasis, while 209 tumors did not show distant metastasis.

Immunohistochemical staining procedure. Sections (4- $\mu \mathrm{m}$ thick) of paraffin and formaldehyde-fixed tissue of the resection specimens were cut. For immunohistochemical staining, sections were deparaffinized and rehydrated via serial passage through xylene and a graded series of ethanol. To block endogenous peroxidase activity slices were treated with $0.3 \% \mathrm{H}_{2} \mathrm{O}_{2}$ for $10 \mathrm{~min}$. All specimens were subjected to heatinduced antigen retrieval in Target Retrieval Solution (Dako Diagnostics, Hamburg, Germany) for $30 \mathrm{~min}$ at $95-99^{\circ} \mathrm{C}$ and then incubated with $10 \%$ normal goat serum to block nonspecific binding. Sections were incubated with the primary c-Met antibody (Santa Cruz Biotechnology, Santa Cruz, CA, USA) at a final dilution of $1: 50$ at $4^{\circ} \mathrm{C}$ overnight. After several washing steps, sections were incubated with biotinylated secondary antibody and streptavidin peroxidase (HRP) (both from Dako Diagnostics). Diaminobenzidine chromogen (Dako Diagnostics) was used for visualization. Nuclei were counterstained with hematoxylin.

Quantitation of immunostaining results. All sections were immunostained under the same conditions in order to reduce the variability. Immunostaining was visualized using a Leica CTR MIC microscope and recorded with a high-resolution DC300 Leica digital camera (Leica Microsystems, Wetzlar, Germany). Five fields (under a x40 objective) from each OSCC section were randomly selected for assessment. After
A

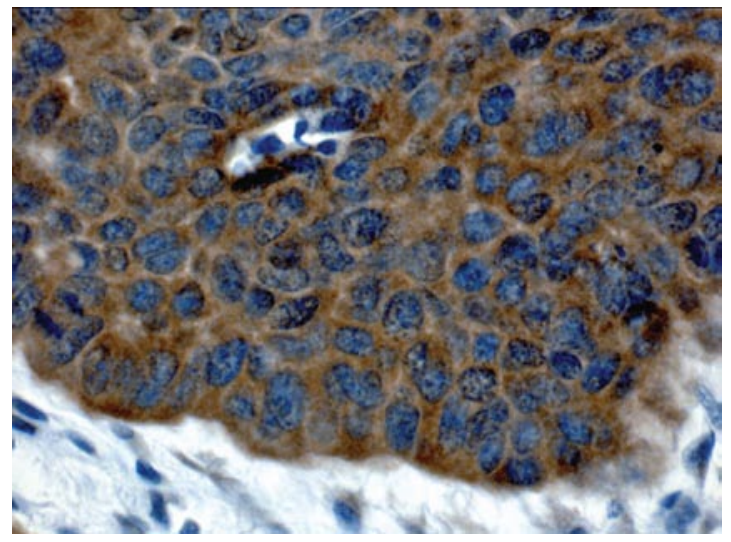

B

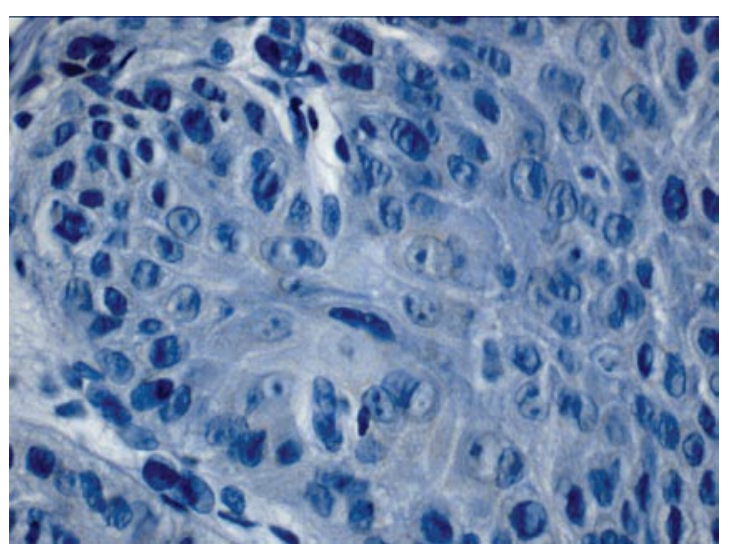

Figure 1. c-Met expression in representative areas of tumors in the c-Metpositive group (percentage of c-Met-expressing cells $\geq 50 \%$ ) (A) and in the c-Met-negative group (percentage of c-Met-expressing cells <50\%) (B) at x40 magnification. c-Met staining was mostly confined to the cytoplasm.

conversion to grayscale images (gray values from 0-255), the c-Met-positive cells were quantified as the percentage of the total cells in five representative view areas using the Leica QWin image analysis and image processing software (Leica Microsystems).

Statistical analysis. Statistical analysis was performed using the Fisher's exact test. A p-value $<0.05$ was indicative of a significant difference. Survival analysis was performed using the the Kaplan-Meier method, and statistical significance was calculated using the log-rank test.

\section{Results}

Immunohistochemical staining was performed against c-Met, and the tumor was defined as negative when antigen expression was demonstrated in $<50 \%$ of the carcinoma cells and as positive when demonstrated in $\geq 50 \%$, as suggested elsewhere (13). Of the 211 samples, a positive result of immunohistochemical staining against c-Met was observed in 175 (82.9\%), while $36(17.1 \%)$ showed no positive result. c-Met expression was not observed in stromal cells, being mainly observed in carcinoma cells. In most of the specimens, c-Met was expressed strongly and diffusely in the cytoplasm of carcinoma cells (Fig. 1). The relation between c-Met expression and clinical and histopathological parameters is summarized 
Table I. Statistical analysis of c-Met expression and associated clinicopathologic parameters in OSCC.

\begin{tabular}{lrrr}
\hline & \multicolumn{2}{l}{ Expression of c-Met; $\mathrm{n}(\%)$} & \\
\cline { 2 - 3 } Variable & Negative & Positive & p-value \\
\hline $\mathrm{T}$ & & & \\
$\mathrm{T}_{1}, \mathrm{~T}_{2}(\mathrm{n}=135)$ & $22(16.3)$ & $113(83.7)$ & 0.706 \\
$\mathrm{~T}_{3}, \mathrm{~T}_{4}(\mathrm{n}=76)$ & $14(18.4)$ & $62(81.6)$ & \\
$\mathrm{N}$ & & & \\
$\mathrm{N}_{0}(\mathrm{n}=146)$ & $28(19.2)$ & $118(80.8)$ & 0.242 \\
$\mathrm{~N}_{1}, \mathrm{~N}_{2}(\mathrm{n}=65)$ & $8(12.3)$ & $57(87.7)$ & \\
$\mathrm{M}$ & & & \\
$-\quad(\mathrm{n}=209)$ & $36(17.2)$ & $173(82.8)$ & 0.321 \\
$+(\mathrm{n}=2)$ & $1(50.0)$ & $1(50.0)$ & \\
Stage & & & \\
$\mathrm{I}, \mathrm{II}(\mathrm{n}=77)$ & $7(9.1)$ & $70(90.9)$ & 0.022 \\
III, IV $(\mathrm{n}=134)$ & $29(21.6)$ & $105(78.4)$ & \\
\hline
\end{tabular}

${ }^{a}$ Calculated using the Fisher's exact test.

in Table I. The rate of positive c-Met expression was $83.7 \%$ in patients with tumors $\leq 4 \mathrm{~cm}$ in diameter (stages $T_{1}$ and $T_{2}$ ) and $81.6 \%$ in tumors $>4 \mathrm{~cm}$ in diameter (stages $\mathrm{T}_{3}$ and $\mathrm{T}_{4}$ ), with no statistically significant difference $(p=0.706)$. Positive c-Met expression seemed to be increased in cases of lymph node metastasis $(87.7 \%)$; however, there was no significant difference compared to those without lymph node metastasis (80.8\%) ( $\mathrm{p}=0.242)$. Where there was no distant metastasis, positive c-Met expression was observed in $82.8 \%$ of the cases, while c-Met expression was positive in $50 \%$ of the cases with distant metastasis, without any statistical significance $(\mathrm{p}=0.321)$. Positive $\mathrm{c}-\mathrm{Met}$ expression decreased in stage III and IV carcinomas (78.4\%) compared to stage I and II carcinomas $(90.9 \%)$ in a statistically significant manner $(\mathrm{p}=0.022)$. Survival analysis was performed using the Kaplan-Meier method, and statistical significance was calculated using the log-rank test (Fig. 2). There was no significant correlation between the groups with negative and positive c-Met expression $(\mathrm{p}=0.8244)$.

\section{Discussion}

In several studies, high expression of HGF and c-Met has been demonstrated in various types of human carcinomas including thyroid, pancreas, prostate and head and neck cancer (8). In particular, studies analyzing c-Met expression in relation to clinical and histopathological parameters in OSCC patients have arrived at controversial results. Thus, to date, a definitive conclusion regarding the prognostic value of c-Met expression in OSCC has not been made. Therefore, we investigated c-Met expression in a large number, 211 cases, of OSCC to verify its possible role as a prognostic marker. A positive c-Met expression, or more than $50 \%$ of the tumor cells showing c-Met staining, was noted in 175 specimens $(82.9 \%)$. This result suggests that in most cases of OSCC,

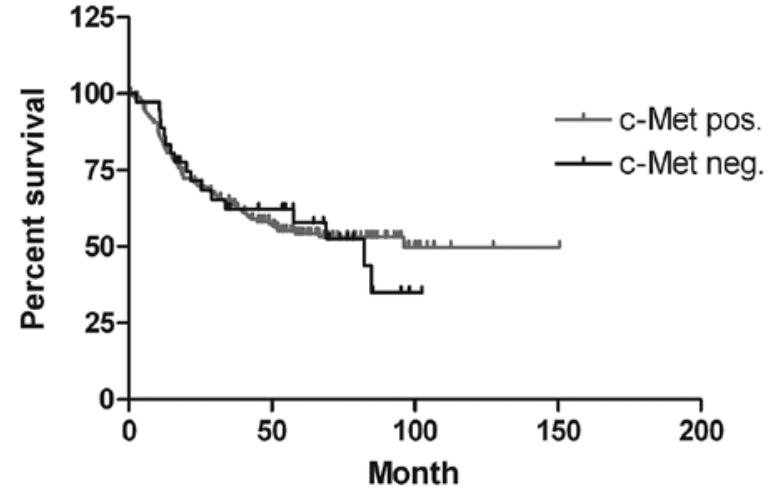

Figure 2. Survival analysis was performed comparing positive (c-Met expression $\geq 50 \%$ ) and negative (c-Met expression $<50 \%$ ) subgroups according to the Kaplan-Meier method, and statistical significance was calculated using the log-rank test. There was no significant difference between both subgroups $(\mathrm{p}=0.8244)$.

c-Met protein is produced. In addition, Kim et al (14), also using immunohistochemical staining, showed that 28 of 40 cases $(70 \%)$ of hypopharyngeal cancer expressed c-Met, and Sawatsubashi et al (15) demonstrated that 45 of 82 cases (54.9\%) of glottic cancer expressed c-Met. Furthermore, c-Met expression was detected in corresponding affected lymph nodes (15-17). In 40 cases of hypopharyngeal squamous cell carcinoma no significant correlation between c-Met expression and tumor size, rate of distant metastasis or survival rate was apparent (14). However, there was a significant increase in c-Met expression in tumors with lymph node metastasis. Sawatsubashi et al (15) reported no significant correlation between c-Met expression and age, gender, histological type, tumor size or distant metastasis in 82 cases of primary laryngeal carcinoma, but 10 of 12 cases $(83.3 \%)$ with lymph node metastasis showed a significant increase in c-Met expression. Lo Muzio et al (3) investigated c-Met expression in 84 patients with OSCC, but no statistical association between c-Met expression and histological grading, lymph node status and staging was found, except there was a significant better survival rate in the group with low c-Met expression than in the group with high c-Met expression. In the present study, using a large sample size of 211 specimens, no significant difference in c-Met expression in stage $T_{1}$ and $T_{2}$ tumors in comparison to stage $T_{3}$ and $T_{4}$ was found. In addition, no significant difference in c-Met expression in cases of lymph node metastasis compared to those without lymph node metastasis could be shown. Furthermore, no statistically significant correlation between the expression of c-Met and survival rate was apparent. Notably, there was a negative significant correlation between clinical tumor stage and c-Met expression.

There are different possible explanations for these inconsistent results. First of all, in most studies, only small numbers of specimens were used to investigate c-Met expression in HNSCC. Kim et al used 40 specimens of hypopharyngeal squamous cell carcinoma (14), Lo Muzio et al used 84 specimens of OSCC (3), Sawatsubashi et al investigated 82 patients with laryngeal carcinomas (15) and Marshall and Kornberg used only 27 samples of OSCC (18). On the other hand, in studies where immunohistochemical 
staining was used, various standards were used to determine a 'positive' result. In one study, expression in $>10 \%$ of cancer cells was considered positive (19), where in other studies c-Met expression in $>30 \%$ of cancer cells was deemed a positive result $(3,14)$. Consequently, we defined a positive result only when expression of c-Met was detected in $\geq 50 \%$ of the cancer cells. Otherwise, human visual assessment of immunohistochemical staining results is difficult and subjective, as the counting results may be varied for each observer and between different observers. The results of quantitative counting and semi-quantitative counting may also be affected by the experience and other psychological factors of the pathologists. To exclude these factors, we objectively quantified the staining result using the Leica QWin image analysis and image processing software.

However, the important role of the HGF/c-Met receptor tyrosine kinase system in the invasive behavior of HNSCC is undisputable, as shown by means of invasion assays in vitro $(20,21)$. Furthermore, a relationship between the concentration of HGF in serum or in cancer tissue and the progression of disease has been noted in patients with gastric, breast and lung cancer (22). Uchida et al (10) reported that fibroblasts of the oral cavity secrete various growth factors, including HGF, which promote the invasion and metastasis of oral cancer cells. In addition, significantly higher HGF serum levels were detected in patients with OSCC when compared with those in healthy volunteers. Furthermore, after initial treatment, all of the tumor-free survivors exhibited a decrease in the serum HGF levels. The exact mechanism of the interaction between HGF and c-Met has not been fully understood. Various cytokines including interleukin-1 and -6 , tumor necrosis factor- $\alpha$, which are produced in the microenvironment of the tumor as a result of the nonspecific inflammatory response, appear to increase c-Met expression (23). Furthermore, c-Met expression was shown to be upregulated by HGF itself, as demonstrated in vitro by Boccaccio et al (24).

In summary, the immunohistochemical staining of oral squamous cell cancer tissue of 211 patients revealed high expression of c-Met. However, the level of c-Met expression showed no significant correlation in relation to tumor size, lymph node status, rate of distant metastasis or survival rate. Moreover, c-Met showed a significant negative correlation to the clinical stage of the tumor. Even though the HGF/c-Met system appears to play an important role in the invasive behavior of OSCC, this study did not verify the role of c-Met as a prognostic marker in patients with OSCC.

\section{References}

1. Lothaire P, De Azambuja E, Dequanter D, et al: Molecular markers of head and neck squamous cell carcinoma: promising signs in need of prospective evaluation. Head Neck 28: 256-269, 2006.

2. De Herdt MJ and Baatenburg De Jong RJ: HGF and c-MET as potential orchestrators of invasive growth in head and neck squamous cell carcinoma. Front Biosci 13: 2516-2526, 2008.

3. Lo Muzio L, Farina A, Rubini C, et al: Effect of c-Met expression on survival in head and neck squamous cell carcinoma. Tumour Biol 27: 115-121, 2006.
4. Bottaro DP, Rubin JS, Faletto DL, et al: Identification of the hepatocyte growth factor receptor as the c-met proto-oncogene product. Science 251: 802-804, 1991.

5. Birchmeier C and Gherardi E: Developmental roles of HGF/SF and its receptor, the c-met tyrosine kinase. Trends Cell Biol 8: 404-410, 1998.

6. Nakamura T, Nishizawa T, Hagiya M, et al: Molecular cloning and expression of human hepatocyte growth factor. Nature 342: 440-443, 1989.

7. Zarnegar R and Michalopoulos GK: The many faces of hepatocyte growth factor: from hepatopoiesis to hematopoiesis. J Cell Biol 129: 1177-1180, 1995.

8. Chen YS, Wang JT, Chang YF, et al: Expression of hepatocyte growth factor and c-met protein is significantly associated with the progression of oral squamous cell carcinoma in Taiwan. $\mathrm{J}$ Oral Pathol Med 33: 209-217, 2004.

9. To $\mathrm{C}$ and Tsao M: The roles of hepatocyte growth factor/scatter factor and met receptor in human cancers (Review). Oncol Rep 5: 1013-1024, 1998.

10. Uchida D, Kawamata H, Omotehara F, et al: Role of HGF/c-met system in invasion and metastasis of oral squamous cell carcinoma cells in vitro and its clinical significance. Int J Cancer 93: 489-496, 2001

11. Matsumoto K, Nakamura T and Kramer RH: Hepatocyte growth factor/scatter factor induces tyrosine phosphorylation of focal adhesion kinase (p125(FAK)) and promotes migration and invasion by oral squamous cell carcinoma cells. J Biol Chem 269: 31807-31813, 1994.

12. Patel SG and Shah JP: TNM staging of cancers of the head and neck: striving for uniformity among diversity. CA Cancer J Clin 55: 242-258, 2005

13. Kunkel M, Reichert T, Benz P, et al: Overexpression of Glut-1 and increased glucose metabolism in tumors are associated with a poor prognosis in patients with oral squamous cell carcinoma. Cancer 97: 1015-1024, 2003.

14. Kim CH, Moon SK, Bae JH, et al: Expression of hepatocyte growth factor and c-Met in hypopharyngeal squamous cell carcinoma. Acta Otolaryngol 126: 88-94, 2006.

15. Sawatsubashi M, Sasatomi E, Mizokami H, Tokunaga O and Shin T: Expression of c-Met in laryngeal carcinoma. Virchows Arch 432: 331-335, 1998.

16. Galeazzi E, Olivero M, Gervasio FC, et al: Detection of MET oncogene/hepatocyte growth factor receptor in lymph node metastases from head and neck squamous cell carcinomas. Eur Arch Otorhinolaryngol 254: S138-S143, 1997.

17. Lo Muzio L, Leonardi R, Mignogna MD, et al: Scatter factor receptor (c-Met) as possible prognostic factor in patients with oral squamous cell carcinoma. Anticancer Res 24: 1063-1069, 2004.

18. Marshall DD and Kornberg LJ: Overexpression of scatter factor and its receptor (c-met) in oral squamous cell carcinoma. Laryngoscope 108: 1413-1417, 1998.

19. Nakajima M, Sawada H, Yamada Y, et al: The prognostic significance of amplification and overexpression of c-met and c-erb B-2 in human gastric carcinomas. Cancer 85: 1894-1902, 1999.

20. Hanzawa M, Shindoh M, Higashino F, et al: Hepatocyte growth factor upregulates E1AF that induces oral squamous cell carcinoma cell invasion by activating matrix metalloproteinase genes. Carcinogenesis 21: 1079-1085, 2000.

21. Murai M, Shen X, Huang L, et al: Overexpression of c-met in oral SCC promotes hepatocyte growth factor-induced disruption of cadherin junctions and invasion. Int J Oncol 25: 831-840, 2004.

22. Han SU, Lee JH, Kim WH, Cho YK and Kim MW: Significant correlation between serum level of hepatocyte growth factor and progression of gastric carcinoma. World J Surg 23: 1176-1180, 1999.

23. Moghul A, Lin L, Beedle A, et al: Modulation of c-MET protooncogene (HGF receptor) mRNA abundance by cytokines and hormones: evidence for rapid decay of the $8 \mathrm{~kb}$ c-MET transcript. Oncogene 9: 2045-2052, 1994.

24. Boccaccio C, Gaudino G, Gambarotta G, Galimi F and Comoglio P: Hepatocyte growth factor (HGF) receptor expression is inducible and is part of the delayed-early response to HGF. J Biol Chem 269: 12846-12851, 1994. 